\title{
Monolithic Fabrication of InSb-based Photo-Pixel for Mid-IR Imaging
}

\author{
Chengzhi Xie ${ }^{1}$, Vincenzo Pusino ${ }^{1}$, Ata Khalid ${ }^{1}$, Mohsin Aziz ${ }^{1}$, Matthew J. Steer ${ }^{1}$,and David R. S. Cumming ${ }^{1}$ \\ ${ }^{1}$ Electronics and Nanoscale Division in the School of Engineering at the University of Glasgow, Glasgow G12 8LT, U.K. \\ Email: c.xie.1@ research.gla.ac.uk
}

\begin{abstract}
We present the monolithic fabrication of an active photo-pixel made in InSb on a GaAs substrate that is suitable for large-scale integration into a focal plane array. Pixel addressing is provided by the co-integration of a GaAs MESFET with an InSb photodiode. Various highly selective etch processes for distinct material layers were established. A low temperature annealed Ohmic contact was also achieved so that the processing temperature never exceeded $180{ }^{\circ} \mathrm{C}$ and no damage to the InSb detectors was observed. Furthermore, since there is a considerable etch step (> $6 \mu \mathrm{m}$ ) that metal must straddle without breaking, we developed an intermediate step using polyimide to provide a smoothing section between the lower MESFET and upper photodiode regions of the device. This heterogeneous technology creates great potential to realize a new type of monolithic focal plane array of addressable pixels for imaging in the medium wavelength infrared range.
\end{abstract}

Keywords-Monolithic integration, medium wavelength infrared photodetector, GaAs, InSb, imaging, focal plane array.

\section{INTRODUCTION AND SUMMARY}

In recent years, a lot of effort has been made to develop imaging capability at medium wavelength infrared (MWIR) or mid-IR range which would significantly contribute to wide range of applications such as gas sensing, security, defense, medical diagnosis, environmental and astronomical observations [1]. In order to make an imaging device, such as a focal plane array (FPA), the detectors must be individually addressable using row and column decoding so that the photo-generated signal can be readout from each pixel. Small band gap semiconductor, such as InSb, is especially suitable for achieving high performance MWIR photodetectors [2]. However, making on-chip readout or signal processing circuit based on this small bandgap semiconductor was found to be extremely difficult and the monolithic imaging sensor made by pure InSb was abandoned after first attempt since 1980s. As a result, hybridization of an array of isolated photodetectors made by small band gap semiconductors with the CMOS addressing chip known as a read-out integrated circuit (ROIC) becomes almost the only choice for state of art MWIR FPAs. Although this hybridization method has successfully produced very large format FPAs, the reliability of interconnections (indium bump bonds) and substrate alignment that used in flip-chip bonding become more challenging when larger arrays of smaller pixels are desired for high resolution cameras at lower cost [3]. Recently, InSb photodetectors have been grown on a GaAs substrate by molecular beam epitaxy (MBE) and reported to have comparable performance to the devices grown on more expensive pure InSb substrates [4]. In addition to providing a cost saving substrate, GaAs can be used as a functional material to fabricate transistors and realize an addressing circuit for the photodetectors.

We present a monolithically integrated switchable photo-pixel which hybridizes an InSb photodiode that is sensitive in the MWIR with a GaAs MESFET by heterogeneous growth of GaAs and InSb device layers on a semi-insulating (SI) GaAs substrate [5] (shown in Fig. 1). As shown in Fig. 2 and Fig. 3, the characterization results confirmed that the fabricated photo-pixel was sensitive to mid-IR range at room temperature, and that the photo-response could be eliminated and isolated from its contacts by switching off the co-integrated MESFET.

In this paper, we will detail more material processing techniques of the InSb-based photo-pixels as can be seen in Fig. 4. The integration method used in this work can be easily migrated to other III-V materials with SI GaAs substrate growth capabilities such as InAsSb and type-II InAs/GaSb superlattices [6][7], therefore alternative semiconductor layer structures could also be investigated. This heterogeneous technology creates great potential to realize a new type of monolithic focal plane array for imaging in the MWIR range without the need for flip-chip bonding to a ROIC.

\section{REFERNCE}

[1] A. Krier, Mid-infrared semiconductor optoelectronics. London: Springer, 2006.

[2] A. Rogalski, "Infrared detectors: an overview", Infrared Physics \& Technology, vol. 43, no. 3-5, pp. 187-210, 2002.

[3] X. Zhang, Q. Meng, L. Zhang and Y. Lv, "Modeling and deformation analyzing of InSb focal plane arrays detector under thermal shock", Infrared Physics \& Technology, vol. 63, pp. 28-34, 2014.

[4] E. Camargo, K. Ueno, Y. Kawakami, Y. Moriyasu, K. Nagase, M. Satou, H. Endo, K. Ishibashi and N. Kuze, "Miniaturized InSb photovoltaic infrared sensor operating at room temperature", Optical Engineering, vol. 47, no. 1, p. 014402, 2008.

[5] C. Xie, V. Pusino, A. Khalid, M. J. Steer, M. Sorel, I. G. Thayne, and D. R. S. Cumming, "Monolithic Integration of an Active InSbbased Mid-Infrared Photopixel With a GaAs MESFET", IEEE Transactions on Electron Devices, vol. 62, no. 12, Dec. 2015.

[6] A. Craig, A. Marshall, Z. Tian, S. Krishna and A. Krier, "Mid-infrared InAs $\mathrm{A}_{0.79} \mathrm{Sb}_{0.21}$-based nBn photodetectors with $\mathrm{Al}_{0.9} \mathrm{Ga}_{0.2} \mathrm{As}_{0.1} \mathrm{Sb}_{0.9}$ barrier layers, and comparisons with $\mathrm{InAs}_{0.87} \mathrm{Sb}_{0.13} \mathrm{p}$-i-n diodes, both grown on GaAs using interfacial misfit arrays", Applied Physics Letters, vol. 103, no. 25, p. 253502, 2013.

[7] Z. Tian, T. Schuler-Sandy, S. Krishna, D. Tang and D. Smith, "Molecular beam epitaxy growth of antimony-based mid-infrared interband cascade photodetectors", Journal of Crystal Growth, vol. 425, pp. 364-368, 2015. 


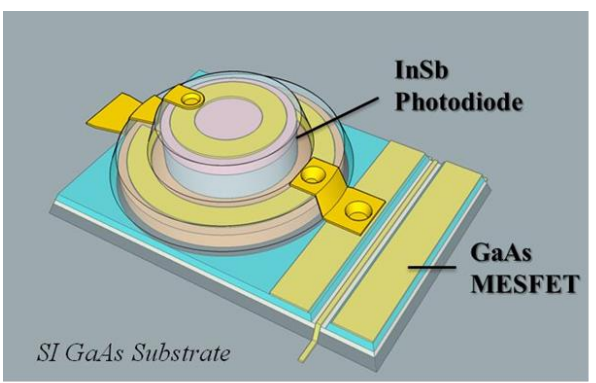

(a)

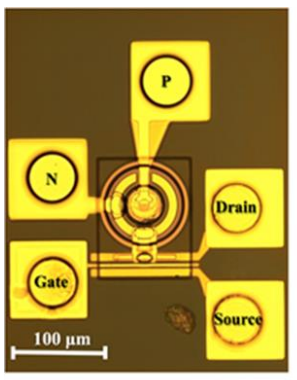

(b)

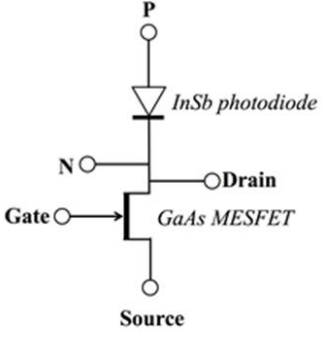

(c)

Fig. 1. (a) A 3D sketch of the monolithically fabricated GaAs MESFET and InSb MWIR detector (b) an optical micrograph and (c) equivalent circuit diagram of an integrated photo-pixel device.

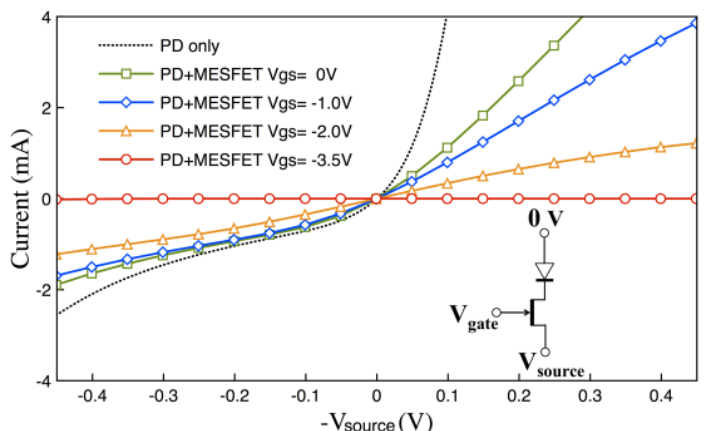

Fig. 2. The current-voltage characteristics of a photo-pixel device with a $45 \mu \mathrm{m}$ diameter circular InSb photodiode.

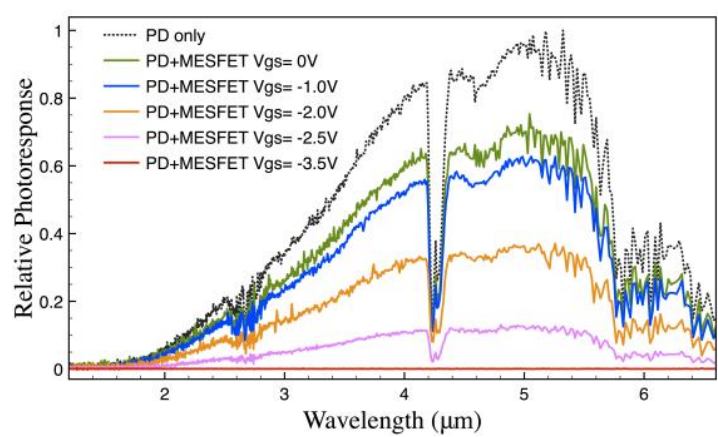

Fig. 3. The relative spectral response of the photo-pixel device under various gate bias conditions.

(a)
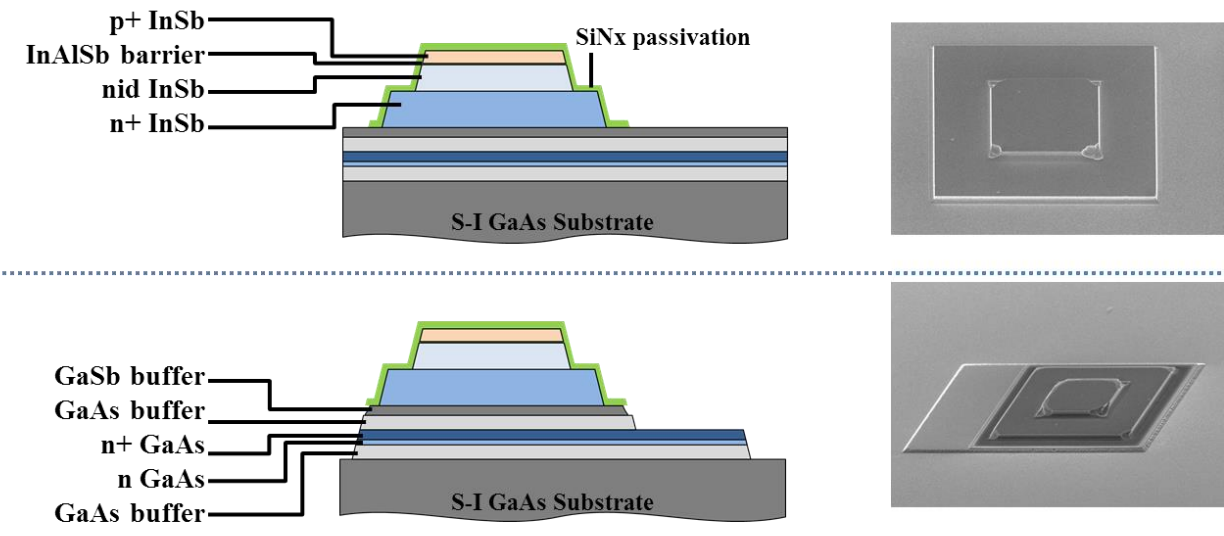

(b) S-I GaAs Substrate

(c)
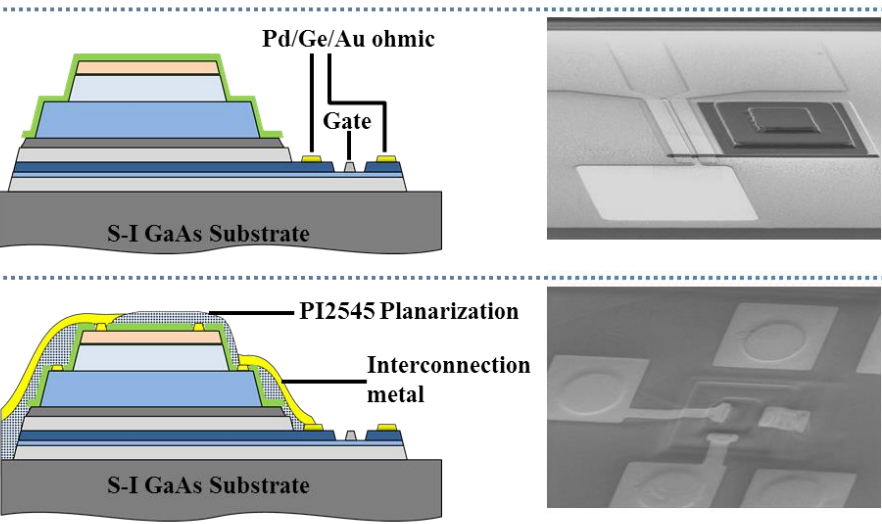

Fig. 4. Fabrication flow of the photo-pixel device (a) define InSb photodiode active area (b) expose the GaAs device layers and isolation etching (c) GaAs MESFET fabrication (d) metal contact to InSb photodiode and interconnection between devices. 